CHIBA-EP-61-REV

\title{
Multicritical Points in a Lattice Yukawa Model with the Wilson-Yukawa Coupling *
}

\author{
Toru Ebihara ${ }^{\dagger}$ and Kei-ichi Kondo $\ddagger$ \\ $\dagger$ Graduate School of Science and Technology, Chiba University, \\ 1-33 Yayoi-cho, Inage-ku, Chiba 263, JAPAN \\ $\ddagger$ Department of Physics, Faculty of Science, Chiba University, \\ 1-33 Yayoi-cho, Inage-ku, Chiba 263, JAPAN
}

\begin{abstract}
For a lattice regularized chiral-invariant $S U(2)_{L} \times S U(2)_{R}$ fermion-scalar model with a Yukawa coupling $y$ and a Wilson-Yukawa coupling $w$, we investigate the phase structure and in particular show the existence of the multicritical line, in the strong Yukawa and/or Wilson Yukawa coupling region, at which four phases meet. The result is in good agreement with the Monte Carlo simulation. This analytical result is derived from the effective scalar model obtained by integrating out the fermion field where the action is explicitly obtained from the hopping parameter expansion up to next-to-leading order. For estimates on the correlation function of the scalar field we apply the mean-field method.
\end{abstract}

$7 / 92$

† E-mail address: ebihara@cuphd.chiba-u.ac.jp; f36598@jpnkudpc.bitnet

‡ E-mail address: c32295@jpnkudpc.bitnet

* To be published in Physics Letters B. 
In the standard model of electroweak interactions the Yukawa couplings of quarks and leptons to the Higgs scalar field provide the masses of the elementary fermions. In particular, these masses are generated through the spontaneous symmetry breaking. However the formulation of the Yukawa models on the lattice suffers from the fermion doubling problem [[1]]: the naively regularized Dirac fermion on the lattice describes additional fermion degrees of freedom (doubler fermions) besides one fermion desired in the continuum limit. To avoids this problem in the chiral theories one must remove unwanted doublers in the continuum limit in such a way that the chiral symmetry is preserved on the lattice [[2], [3]]. One formulation of the Yukawa model adds a manifestly chiral invariant Wilson-Yukawa coupling to decouple the doublers from the physical spectrum by rendering them heavy dynamically [[3]]. The other method introduces mirror fermions explicitly, in addition to the original field, which transform oppositely under the chiral symmetry group [4]].

In this letter we examine the former one, lattice Yukawa model with a chiral invariant Wilson-Yukawa term with coupling constant $w$, which reduces to the naive lattice Yukawa model with only a Yukawa coupling $y$ in the limit $w \rightarrow 0$. The naive lattice Yukawa model $(w=0)$ which is defined below has a rich phase structure as shown in fig. 1, which is already discussed in the previous paper [[5]]. To distinguish the phase, two order paramters have been introduced: one is the usual expectation value of the scalar field $\phi_{x}$ and another is the expectation value of the staggered scalar field defined by $\hat{\phi}_{x}:=(-1)^{x_{1}+x_{2}+\cdots+x_{d}} \phi_{x}$ where $d$ denotes the dimension of the Euclidean space. The phase diagram consists of the ferromagnetic $(\mathrm{FM})$ phase $(\langle\phi\rangle \neq 0,\langle\hat{\phi}\rangle=0)$, the paramagnetic $(\mathrm{PM})$ phase $(\langle\phi\rangle=0,\langle\hat{\phi}\rangle=0)$, the antiferromagnetic (AFM) phase $(\langle\phi\rangle=0,\langle\hat{\phi}\rangle \neq 0)$ and the ferrimagnetic (FI) phase $(\langle\phi\rangle \neq 0,\langle\hat{\phi}\rangle \neq 0)$. One of the most characteristic features of this phase diagram is that $\mathrm{PM}$ and AFM phases are separated into their weak $(\mathrm{W})$ and strong coupling regions $(\mathrm{S})$ by the presence of the intermediate FI phase. For $w=0$ the various phase transition lines intersect at the two quadruple points $A$ and $B$ where four phases can meet.

At a nonzero value of the Wilson-Yukawa coupling $w \neq 0$ the phase diagram can be approximately obtained by shifting in fig. 1 the $y=0$ axis by an amount of $d w$ in the positive $y$-direction, as confirmed by various results of numerical simulations [[6]]. This is indeed understood from the leading result of the hopping parameter expansion given below. In the 3 -dimensional bare parameter space $(y, w, \kappa)$ the two points $A$ and $B$ become two lines which we shall again label by $A$ and $B$ respectively.

Quite recently Bock et al. [[7]] have shown that in the weak coupling region including the point $A$ the unwanted fermion doublers can not be removed from the physical particle 
spectrum. This implies that in the weak $w$ region the continuum limit is not appropriate for describing the electroweak standard model. At least for $w=0$, moreover, the existence of a nontrivial fixed point at $\mathrm{A}$ is quite unlikely from the result of the simulation for the renormalized Yukawa coupling [[8]]. Thus the weak coupling region in the neighborhood of the origin $y=w=0$ is governed by the Gaussian fixed point up to the point $A$. For $w=0$, Jülich group [[7]] has also shown that the fermions become massive due to the strong Yukawa coupling $y$ near the point $B$, which prevents the theory from having the meaningful continuum limit. Taking into account these facts, the physically meaningful point in the continuum limit is expected to reside in the vicinity of the multiple (would-be multicritical) point $B$ in the weak naive-Yukawa coupling $y \ll 1$ and strong Wilson-Yukawa coupling $w \sim 1$.

This paper is the first to show by an analytical method that even in the presence of the Wilson-Yukawa coupling there are multicritical points, corresponding to $B$ in the naive case $w=0$, in the strong Yukawa and/or strong Wilson-Yukawa coupling region. This might corresponds to a nontrivial fixed point at which an interacting continuum limit can be realized. Moreover in the presence of the Wilson-Yukawa coupling the doubler mass can get mass of the order of the cutoff and therefore they are removed from the physical spectrum in the continuum limit. Basic ingredients are the hopping parameter expansion in higher orders and the estimates on the expectation value of the scalar fields, of which the mean field is the simplest one.

The chiral $S U(2)_{L} \times S U(2)_{R}$ symmetric Yukawa model on the lattice is defined by the action $S=S_{H}+S_{F}+S_{Y}+S_{W}$ with

$$
\begin{aligned}
S_{H} & =-\kappa \sum_{x, \mu} \frac{1}{2} \operatorname{tr}\left(\Phi_{x}^{\dagger} \Phi_{x+\mu}+\Phi_{x}^{\dagger} \Phi_{x-\mu}\right) \\
S_{F} & =\frac{1}{2} \sum_{x, \mu}\left\{\bar{\psi}_{x} \gamma_{\mu} P_{L}\left(\psi_{x+\mu}-\psi_{x-\mu}\right)+\bar{\psi}_{x} \gamma_{\mu} P_{R}\left(\psi_{x+\mu}-\psi_{x-\mu}\right)\right\} \\
S_{Y} & =y \sum_{x} \bar{\psi}_{x}\left(\Phi_{x} P_{R}+\Phi_{x}^{\dagger} P_{L}\right) \psi_{x} \\
S_{W} & =w \sum_{x, \mu}\left\{\bar{\psi}_{x}\left(\Phi_{x} P_{R}+\Phi_{x}^{\dagger} P_{L}\right) \psi_{x}\right. \\
& \left.-\frac{1}{2} \bar{\psi}_{x}\left(\Phi_{x} P_{R}+\Phi_{x+\mu}^{\dagger} P_{L}\right) \psi_{x+\mu}-\frac{1}{2} \bar{\psi}_{x}\left(\Phi_{x} P_{R}+\Phi_{x-\mu}^{\dagger} P_{L}\right) \psi_{x-\mu}\right\},
\end{aligned}
$$

where $\kappa$ is the hopping parameter for the scalar field, $y$ the normal Yukawa coupling, $w$ the Wilson-Yukawa coupling, and $P_{L}$ and $P_{R}$ are the left- and right-handed chiral projectors, 
$P_{L}=\frac{1}{2}\left(1-\gamma_{5}\right), P_{R}=\frac{1}{2}\left(1+\gamma_{5}\right)$. Here the scalar field $\Phi_{x}$ is radially fixed $\Phi_{x} \Phi_{x}^{\dagger}=1$ and is expressed by $S U(2)$ matrix and the fermion field $\psi$ and $\bar{\psi}$ are $S U(2)$ doublets. The action has the global chiral $S U(2)_{L} \times S U(2)_{R}$ symmetry: $\psi_{x} \rightarrow\left(\Omega_{L} P_{L}+\Omega_{R} P_{R}\right) \psi_{x}$, $\bar{\psi}_{x} \rightarrow \bar{\psi}_{x}\left(\Omega_{L}^{\dagger} P_{L}+\Omega_{R}^{\dagger} P_{R}\right), \Phi_{x} \rightarrow \Omega_{L} \Phi_{x} \Omega_{R}^{\dagger}$, where $\Omega_{L, R} \in S U(2)_{L, R}$ respectively.

It is convenient to transfer into the newly defined fermion field: $\psi_{x}^{\prime}=[y+$ $d w]^{-1 / 2}\left(\Phi_{x}^{\dagger} P_{L}+P_{R}\right) \psi_{x}, \bar{\psi}_{x}^{\prime}=[y+d w]^{-1 / 2} \bar{\psi}_{x}\left(\Phi_{x} P_{R}+P_{L}\right)$. Then the fermionic part of the action (11) is rewritten as follows:

$$
S_{F}+S_{Y}+S_{W}=\sum_{x} \bar{\psi}_{x}^{\prime} \psi_{x}^{\prime}-K \sum_{x, y} \bar{\psi}_{x}^{\prime} Q_{x, y}\left(\Phi, \Phi^{\dagger}\right) \psi_{y}^{\prime}
$$

where the matrix $Q_{x, y}$ is defined by

$$
Q_{x, y}=\sum_{\mu=1}^{d}\left[w-\gamma_{\mu}\left(\Phi_{x}^{\dagger} \Phi_{x+\mu} P_{L}+P_{R}\right)\right] \delta_{y, x+\mu}+\sum_{\mu=1}^{d}\left[w+\gamma_{\mu}\left(\Phi_{x}^{\dagger} \Phi_{x-\mu} P_{L}+P_{R}\right)\right] \delta_{y, x-\mu}
$$

with a hopping parameter

$$
K=\frac{1}{2(y+d w)}
$$

Here we notice that the fermionic part of the action has the bilinear form in the fermion field. After integrating out the fermion field, the model is converted into the effective scalar model with the action:

$$
S_{\mathrm{eff}}=S_{H}-N_{f} \ln \operatorname{Det} M
$$

where $N_{f}$ is the number of fermion species and the matrix $M_{x, y}$ is defined by

$$
M_{x, y}\left(\Phi, \Phi^{\dagger}\right)=\left(\mathbf{1}-K Q\left(\Phi, \Phi^{\dagger}\right)\right)_{x, y}
$$

Now we perform the hopping parameter expansion (HPE) [[9]]. The first step is to expand $\ln$ Det $M$ in powers of the hopping parameter $K$ as follows:

$$
\ln \operatorname{Det} M_{x, y}=\operatorname{Tr} \ln [\mathbf{1}-K Q]_{x, y}=-\sum_{n=1}^{\infty} \frac{1}{2 n} K^{2 n} \sum_{x_{1}, \ldots, x_{2 n}} \operatorname{tr}\left[Q_{x_{1}, x_{2}} \cdots Q_{x_{2 n}, x_{1}}\right]
$$

where tr should be understood over the space of the Dirac spinor and the group $S U(2)$. 
Our result of the HPE up to $O\left(K^{4}\right)$ is

$$
\begin{aligned}
& \ln \text { Det } M=-\frac{1}{2} K^{2} D \sum_{x, \mu}\left(2 w^{2} \operatorname{tr}(1)-2 \operatorname{tr}\left[\Phi_{x}^{\dagger} \Phi_{x+\mu}\right]\right) \\
& -\frac{1}{4} K^{4} D \sum_{x, \mu}\left(2 w^{4}(7 d-4) \operatorname{tr}(1)-12 w^{2} \operatorname{tr}\left[\Phi_{x}^{\dagger} \Phi_{x+\mu}\right]\right) \\
& -\frac{1}{4} K^{4} D \sum_{x, \mu}\left(4 \operatorname{tr}\left[\Phi_{x}^{\dagger} \Phi_{x+\mu} \Phi_{x}^{\dagger} \Phi_{x-\mu}\right]+2 \operatorname{tr}\left[\Phi_{x}^{\dagger} \Phi_{x+\mu} \Phi_{x}^{\dagger} \Phi_{x+\mu}\right]\right) \\
& -\frac{1}{4} K^{4} D \sum_{x, \mu, \nu}\left(8 \operatorname{tr}\left[\Phi_{x}^{\dagger} \Phi_{x+\mu} \Phi_{x+\mu+\nu}^{\dagger} \Phi_{x+\mu}\right]-4 \operatorname{tr}\left[\Phi_{x}^{\dagger} \Phi_{x+\mu} \Phi_{x+\mu+\nu}^{\dagger} \Phi_{x+\nu}\right]\right),
\end{aligned}
$$

where $D=2^{d / 2}$ for even $d$. Here note that the trace is taken over the group $G$ and that this result is independent of the choice of $G$. In the next step we specify the group: $G=S U(2)$. The radially fixed scalar field $\Phi_{x}$ is a $2 \times 2 S U(2)$ matrix and therefore can be written as $\Phi=T_{\alpha} \phi^{\alpha}(\alpha=0,1,2,3)$ and its conjugate as $\Phi^{\dagger}=\bar{T}_{\alpha} \phi^{\alpha}$ where

$$
T_{\alpha}=\left(\sigma_{0}, i \sigma_{m}\right), \bar{T}_{\alpha}=\left(\sigma_{0},-i \sigma_{m}\right),(\alpha=0,1,2,3 ; m=1,2,3)
$$

with a unit $2 \times 2$ matrix $\sigma_{0}$ and Pauli matrices $\sigma_{m}$. By making use of the following identities for traces:

$$
\begin{gathered}
\frac{1}{2} \operatorname{tr}\left[T^{\alpha} \bar{T}^{\beta}\right]=\delta^{\alpha, \beta} \\
\frac{1}{2} \operatorname{tr}\left[T^{\alpha} \bar{T}^{\beta} T^{\rho} \bar{T}^{\sigma}\right]=\delta^{\alpha, \beta} \delta^{\rho, \sigma}-\delta^{\alpha, \rho} \delta^{\beta, \sigma}+\delta^{\alpha, \sigma} \delta^{\beta, \rho}
\end{gathered}
$$

final expression of $\ln$ Det $M$ is obtained as follows.

$$
\begin{aligned}
& \ln \operatorname{Det} M=-D K^{2} \sum_{x, \mu}\left[2 w^{2}-2 \phi_{x}^{\alpha} \phi_{x+\mu}^{\alpha}\right] \\
& -D K^{4} \sum_{x, \mu}\left(w^{4}(7 d-4)-6 w^{2} \phi_{x}^{\alpha} \phi_{x+\mu}^{\alpha}\right) \\
& -D K^{4} \sum_{x, \mu}\left(2 \phi_{x}^{\alpha} \phi_{x+\mu}^{\alpha} \phi_{x}^{\beta} \phi_{x-\mu}^{\beta}-2 \phi_{x+\mu}^{\alpha} \phi_{x-\mu}^{\alpha}+\phi_{x}^{\alpha} \phi_{x+\mu}^{\alpha} \phi_{x}^{\beta} \phi_{x+\mu}^{\beta}-\frac{1}{2}\right) \\
& -D K^{4} \sum_{x, \mu, \nu}\left(4 \phi_{x}^{\alpha} \phi_{x+\mu}^{\alpha} \phi_{x+\mu}^{\beta} \phi_{x+\mu+\nu}^{\beta}-2 \phi_{x}^{\alpha} \phi_{x+\mu+\nu}^{\alpha}\right. \\
& \left.-\phi_{x}^{\alpha} \phi_{x+\mu}^{\alpha} \phi_{x+\nu}^{\beta} \phi_{x+\mu+\nu}^{\beta}+\phi_{x}^{\alpha} \phi_{x+\mu+\nu}^{\alpha} \phi_{x+\mu}^{\beta} \phi_{x+\nu}^{\beta}-\phi_{x}^{\alpha} \phi_{x+\nu}^{\alpha} \phi_{x+\mu}^{\beta} \phi_{x+\mu+\nu}^{\beta}\right) .
\end{aligned}
$$

Thus we have obtained the action of the effective scalar model up to $O\left(K^{4}\right)$. 
In order to estimate the two- and the four-point correlation functions of the scalar field, we apply the simplest mean-field (MF) method. We introduce the external field $H^{\alpha} \quad(\alpha=0,1,2,3)$ and define the expectation $\langle\cdot\rangle_{H}$ by

$$
\begin{aligned}
\langle(\cdot)\rangle_{H} & =Z_{H}^{-1} \int \prod_{x, \alpha} d \phi_{x}^{\alpha} \exp \left(\sum_{x, \alpha} H^{\alpha} \phi_{x}^{\alpha}\right)(\cdot) \\
Z_{H} & =\int \prod_{x, \alpha} d \phi_{x}^{\alpha} \exp \left(\sum_{x, \alpha} H^{\alpha} \phi_{x}^{\alpha}\right) .
\end{aligned}
$$

In this framework of MF theory, we can compute the correlation function explicitly, using the fact:

$$
\left\langle\phi_{x}^{\alpha}\right\rangle_{H}=H^{\alpha} / 4+O\left(H^{3}\right),\left\langle\phi_{x}^{\alpha} \phi_{x}^{\beta}\right\rangle_{H}=\delta^{\alpha, \beta} / 4+O\left(H^{2}\right),
$$

e.g., $\left\langle\Phi_{0}^{\dagger} \Phi_{1} \Phi_{1+2}^{\dagger} \Phi_{1}\right\rangle=$ const. $-\frac{H^{2}}{8}+O\left(H^{3}\right)$. For the MF free energy given by

$$
F(H)=\left\langle S_{\mathrm{eff}}\right\rangle_{H}+\sum_{x, \alpha} H^{\alpha}\left\langle\phi_{x}^{\alpha}\right\rangle_{H}-\ln Z_{H}
$$

the stability-violating condition of the PM phase [[50] $\left.\frac{\partial^{2}}{\partial H^{2}} F(H)\right|_{H=0}=0$, yields the critical surface separating the FM phase from the PM phase in the three-dimensional bare parameter space $(y, w, \kappa)$ as follows. $F M(S)-P M(S)$ critical surface:

$$
\kappa_{1}=\frac{2}{d}\left[1-\tilde{N}_{f}\left(K^{2} d+K^{4}\left(6 d^{2}-3 d\right) w^{2}+\frac{1}{2} K^{4}\left(2 d^{2}-d\right)\right)\right],
$$

where $\tilde{N}_{f}=N_{f} D=N_{f} 2^{d / 2}$. To obtain the critical surface separating the AFM phase from the PM phase, on the other hand, we have only to evaluate $\ln$ Det $M$ in terms of the staggered field $\hat{\Phi}$. Using the translational invariance, we obtain

$$
\begin{aligned}
& \langle\ln \operatorname{Det} M\rangle_{H}=-\frac{1}{2} K^{2} D V d\left(w^{2} \operatorname{tr}(1)+\left\langle\operatorname{tr} \hat{\Phi}_{0}^{\dagger} \hat{\Phi}_{1}\right\rangle_{H}\right) \\
& -\frac{1}{4} K^{4} D V\left\{2 d(7 d-4) w^{4} \operatorname{tr}(1)+12 d(2 d-1) w^{2}\left\langle\operatorname{tr} \hat{\Phi}_{0}^{\dagger} \hat{\Phi}_{1}\right\rangle_{H}\right. \\
& +8 d(d-1)\left\langle\operatorname{tr} \hat{\Phi}_{0}^{\dagger} \hat{\Phi}_{1} \hat{\Phi}_{1+2}^{\dagger} \hat{\Phi}_{1}\right\rangle_{H}-4 d(d-1)\left\langle\operatorname{tr} \hat{\Phi}_{0}^{\dagger} \hat{\Phi}_{1} \hat{\Phi}_{1+2}^{\dagger} \hat{\Phi}_{2}\right\rangle_{H} \\
& \left.+4 d\left\langle\operatorname{tr} \hat{\Phi}_{0}^{\dagger} \hat{\Phi}_{1} \hat{\Phi}_{1+1}^{\dagger} \hat{\Phi}_{1}\right\rangle_{H}+2 d\left\langle\operatorname{tr} \hat{\Phi}_{0}^{\dagger} \hat{\Phi}_{1} \hat{\Phi}_{0}^{\dagger} \hat{\Phi}_{1}\right\rangle_{H}\right]+O\left(K^{6}\right),
\end{aligned}
$$

where $V$ is the volume of the lattice. Applying the MF theory similarly, we obtain from the above condition $A F M(S)-P M(S)$ critical surface:

$$
\kappa_{2}=\frac{2}{d}\left[-1-\tilde{N}_{f}\left(K^{2} d+K^{4}\left(6 d^{2}-3 d\right) w^{2}-\frac{1}{2} K^{4}\left(2 d^{2}-d\right)\right)\right] .
$$


Now we pay attention to the intersection point of the two critical surfaces $\kappa=\kappa_{1}$, $\kappa=\kappa_{2}$ just obtained. In the leading order $\left(O\left(K^{2}\right)\right)$ of HPE, there is no cross point as in the case of $w=0$ [[5], [10]]. In the next-to-leading order $\left(O\left(K^{4}\right)\right)$, the cross point does appear and makes a line in the bare parameter space $(y, w, \kappa)$, see fig. 2 (a). This is nothing but the multiple point at which four different phases meet. By equating both equation for the critical surface, $\kappa_{1}=\kappa_{2}$, the intersection line $\kappa=\kappa_{c}$ is obtained:

$$
y+d w=\frac{1}{2}\left[\frac{\tilde{N}_{f}}{2}\left(2 d^{2}-d\right)\right]^{1 / 4}
$$

which is depicted in fig. 2 (b). Indeed, in the limit $w \rightarrow 0$, this line has the end-point, $B=\left(y_{B}, 0, \kappa_{B}\right): y_{B}=\frac{1}{2}\left[\frac{\tilde{N}_{f}}{2}\left(2 d^{2}-d\right)\right]^{1 / 4}, \kappa_{B}=-\frac{2}{d}\left[\frac{2 \tilde{N}_{f} d}{2 d-1}\right]^{1 / 2}$, which agrees with the previous result [[5]]. Another end-point in the limit $y=0$ locates at $B^{\prime}=\left(0, w_{B^{\prime}}, \kappa_{B^{\prime}}\right)$ : $w_{B^{\prime}}=\frac{1}{2 d}\left[\frac{\tilde{N}_{f}}{2}\left(2 d^{2}-d\right)\right]^{1 / 4}, \kappa_{B^{\prime}}=-\frac{2}{d}\left[\frac{2 d \tilde{N}_{f}}{2 d-1}\right]^{1 / 2}\left\{1+\frac{3}{4 d^{2}}(2 d-1)\right\}$, where $w_{B^{\prime}}=0.48$, $\kappa_{B^{\prime}}=-2.84$ for $N_{f}=4, d=4$.

Although our explicit calculation in the presence of the Wilson-Yukawa term $(w \neq 0)$ is limited up to order $K^{4}$, the existence of the multicritical line is guaranteed up to order $O\left(K^{8}\right)$, since this line should have in the limit $w=0$ the cross point $B$ whose existence is confirmed up to $O\left(y^{-8}\right)$ in the previous paper [[5]]. Strictly speaking, we can not exclude the case that the multicritical line may terminate for large $w$ and small $y$. However the Monte Carlo simulations [ [6]] show that this is not the case.

An important aspect of lattice construction which is not mentioned in this paper concerns the reflection positivity $(\mathrm{RP})$. So far RP could be proven in a restricted range of the bare parameters in a restricted class of models [[1] , [12]]. It is not yet clear whether RP holds also in the model treated in this paper [[13]] and in particular in the region which we are focusing on.

In conclusion, we have shown by the analytical method based on the hopping parameter expansion up to next-to-leading order that the multicritical line at which four phases meet exist even in the presence of the Wilson-Yukawa coupling $w$ for the chiral $S U(2)_{L} \times S U(2)_{R}$ symmetric lattice Yukawa model. This multicritical line is connected to the multicritical point $B$ in the naive Yukawa model $w=0$. In other words, the $\operatorname{PM}(\mathrm{W})$ phase and the $\mathrm{PM}(\mathrm{S})$ phase are separated by the intermediate FI phase. As a result it turns out that the $\mathrm{PM}(\mathrm{W})$ phase is not analytically connected to the $\mathrm{PM}(\mathrm{S})$ phase, and the physics in these two respective phases may be rather different. 
In our model the scalar field is radially fixed which corresponds to the infinite selfcoupling limit $\lambda \rightarrow \infty$ of the scalar potential, $\lambda\left(\Phi \Phi^{\dagger}-1\right)^{2}$. It is a common wisdom that the radially fixed scalar model belongs to the same universality class as the weak-coupling one in the pure scalar sector. However recent numerical result based on large $N$ shows that at small bare quartic self-couplling $\lambda$ the point $A$ is replaced by a first order phase transition line, before the FM and AFM phases come close to each other [[14]]. Therefore the multicritical point $B^{\prime}$ in the limit $y \rightarrow 0$ is expected to be only one possible point for a nontrivial ultraviolet fixed point, since the vanishing of the fermion mass in lattice units in the limit is guaranteed from the Golterman-Petcher symmetry [ [15]] and the doublers are removed by giving them masses of the order of the cutoff.

There are however recent works [[16], [17], [18]] which claim that the continuum limit $\kappa \rightarrow \kappa_{c}, y \rightarrow 0$ from the strong coupling regions $\operatorname{FM}(\mathrm{S})$ and $\mathrm{PM}(\mathrm{S})$ does most probably not lead to the desired formulation of the scalar-fermion sector of the Standard model on the lattice. It has been demonstrated in these works that the spectrum in the $\operatorname{PM}(\mathrm{S})$ phase consists only of the scalar particles $(\Phi)$ and a neutral fermion described by the field $\psi^{\prime}$. Moreover it has been argued that one ends up with an almost non-interacting neutral fermion in the continuum limit from the $\mathrm{FM}(\mathrm{S})$ phase [[16]], since the renormalized Yukawa coupling of the neutral fermion to the scalar particles vanishes as a power of the lattice spacing $a$. In this scenario the dimensionless scalar expectation $\left\langle\Phi_{r e n}\right\rangle$ goes to zero in the continuum limit.

However we do not agree with this scenario and expect another one. In order to get the theory in the broken-symmetry phase by taking the scaling continuum limit, bare parameters must be adjusted in such a way that the vacuum expectation value of the renormalized scalar field $\left\langle\Phi_{r e n}\right\rangle$ take a non-zero (finite) value in the limit. To perform this actually, we need detailed information on the phase structure and the critical behavior of the model as suggested from experiences in the scalar $\lambda \phi^{4}$ theory. The scalar $\lambda \phi^{4}$ theory in the broken-symmetry phase constructed in the above sense has no self-interactions, i.e., the (dimensionless) renormalized three- and four-point self-couplings vanish in the continuum limit [[19]] . This is nothing but the triviality of the broken-symmetry $\lambda \phi^{4}$ theory. Thus we expect a scenario in which the non-vanishing Yukawa coupling is obtained by keeping the renormalized expectation value non-zero in the scaling limit by giving the triviality of the pure scalar sector as a sacrifice. We do not think that the triviality of the Yukawa coupling has been established at least in the case of approaching the line B-B' from the 
broken-symmetry phase. Detailed discussion on this issue will be given in a subsequent paper [[20]].

The behavior of the scalar propagator has already been investigated in the neighborhood of two multicritical points [[8]]. As a result, it has been shown that the scalar particle spectrum near the multicritical points A and B contains beside the usual bosonic particle additional 'staggered' particles which emerge as a consequence of the antiferromagnetic ordering at negative $\kappa$. The emergence of these spurious particles and the antiferromagnetic phases has to be simply regarded as an artifact of the special hypercubic lattice geometry and is a non-universal feature of this special kind of lattice regularization. However we do not think that the existence of two multicritical points A and B may be such a lattice artifact. This issue will deserve further studies.

\section{Acknowledgements}

The authors would like to thank the referee for recalling and informing them recent works mentioned in the discussion. 


\section{References}

[1] H.B. Nielsen and M. Ninomiya, Nucl. Phys. B185 (1981) 20, ibid. B185 (1981) 173.

[2] S. Aoki, Phys. Rev. Lett. 60 (1988) 2109; K. Funakubo and T. Kashiwa, ibid. 60 (1988) 2113.

[3] J. Smit, Acta Physica Polonica B17 (1986) 531; P.V.D. Swift, Phys. Lett. B145 (1984) 256.

[4] I. Montvay, DESY preprint, DESY-92-001.

[5] T. Ebihara and K.-I. Kondo, Prog. Theor. Phys. 89 (1992) 1019.

[6] W. Bock, A.K. De, K. Jansen, J. Jersák, T. Neuhaus and J. Smit, Nucl. Phys. B344 (1990) 207.

[7] W. Bock, A.K. De, C. Frick, K. Jansen and T. Trappenberg, Nucl. Phys. B371 (1992) 683.

[8] W. Bock, A.K. De, C. Frick, J. Jersák and T. Trappenberg, HLRZ-91-82/ITFA-91-33;

C. Frick, talk given at the Conf. Lattice'91, Tsukuba, Japan.

[9] S. Aoki, I-H. Lee and R.E. Shrock, Nucl. Phys. B355 (1991) 383.

[10] M.A. Stephanov and M.M. Tsypin, Phys. Lett. B261 (1991) 109.

[11] L. Lin, I. Montvay, G. Münster and H. Wittig, Nucl. Phys. B355 (1991) 511.

[12] S.V. Zenkin, DFTUZ/92/7.

[13] J. Smit, Nucl. Phys. B (Proc. Suppl.) 20 (1991) 542.

[14] A. Hasenfratz, K. Jansen and Y. Shen, talk given at Lattice'91.

[15] M.F.L. Golterman and D.N. Petcher, Phys. Lett. B225 (1989) 159.

[16] M.F.L. Golterman, D.N. Petcher and J. Smit, Nucl. Phys. B370 (1993) 51; D.N. Petcher, talk given at the Conf. Lattice'91, Tsukuba, Japan.

[17] M.F.L. Golterman, D.N. Petcher and E. Rivas, Wash. U. HEP/91-82.

[18] W. Bock, A.K. De and J. Smit, HLRZ-91-81/ITFA-91-30; W. Bock, talk given at the Conf. Lattice'91, Tsukuba, Japan.

[19] K.-I. Kondo, Prog. Theor. Phys. 79 (1988) 1217 and references therein.

[20] T. Ebihara and K.-I. Kondo, in preparation. 


\section{Figure Captions}

Fig. 1. Phase diagram of the naive Yukawa model $(w=0)$ for $N_{f}=4, d=4$.

Fig. 2. Phase diagram of the Yukawa model with the Wilson-Yukawa term for $N_{f}=4$, $d=4$ :

(a) schematic three-dimensional phase diagram,

(b) projection of the phase diagram on the $\kappa=$ constant plane . 


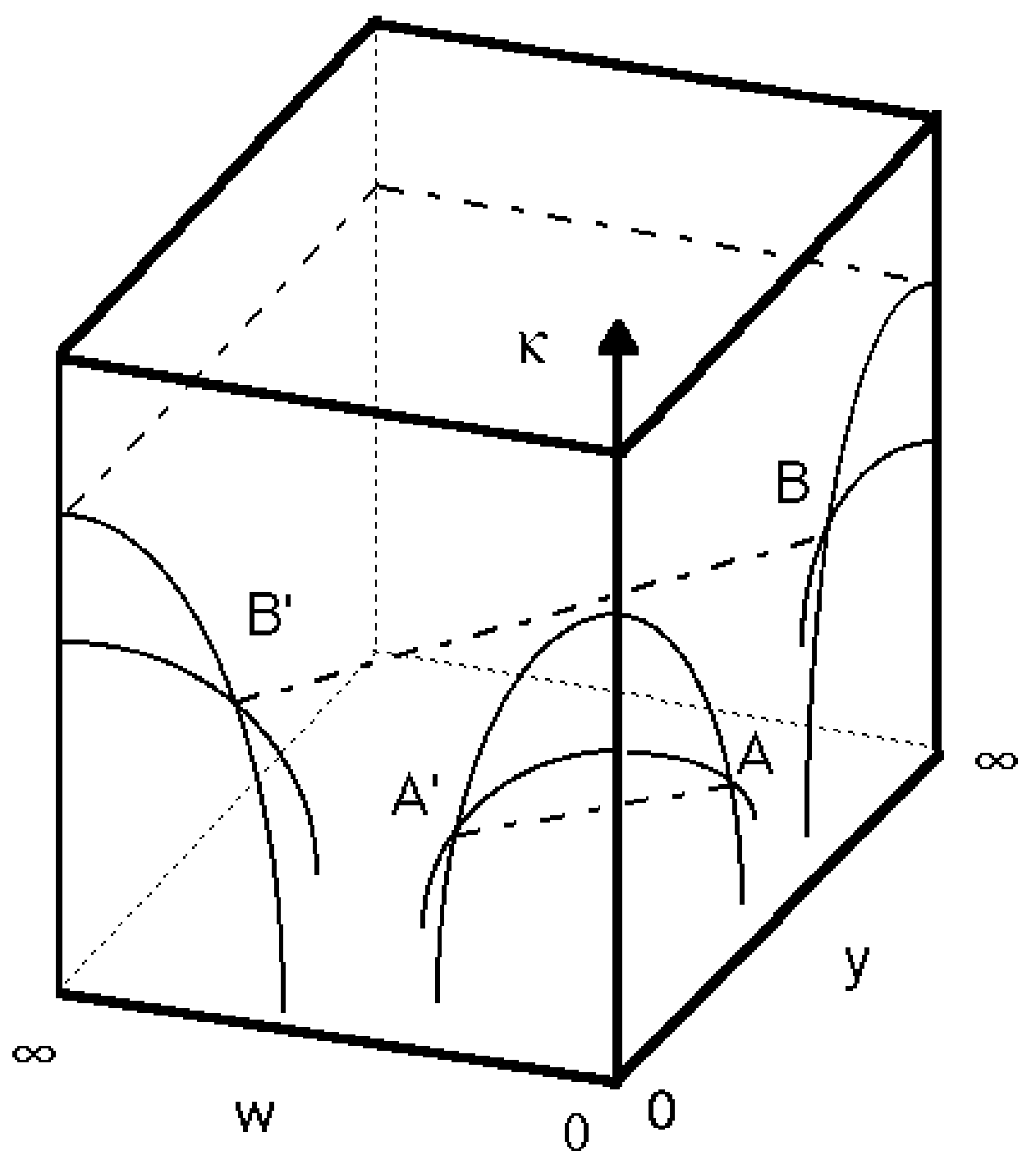

Fig. 2(a): Schematic three-dimensional phase diagram with the Wilson-Yukawa term. 


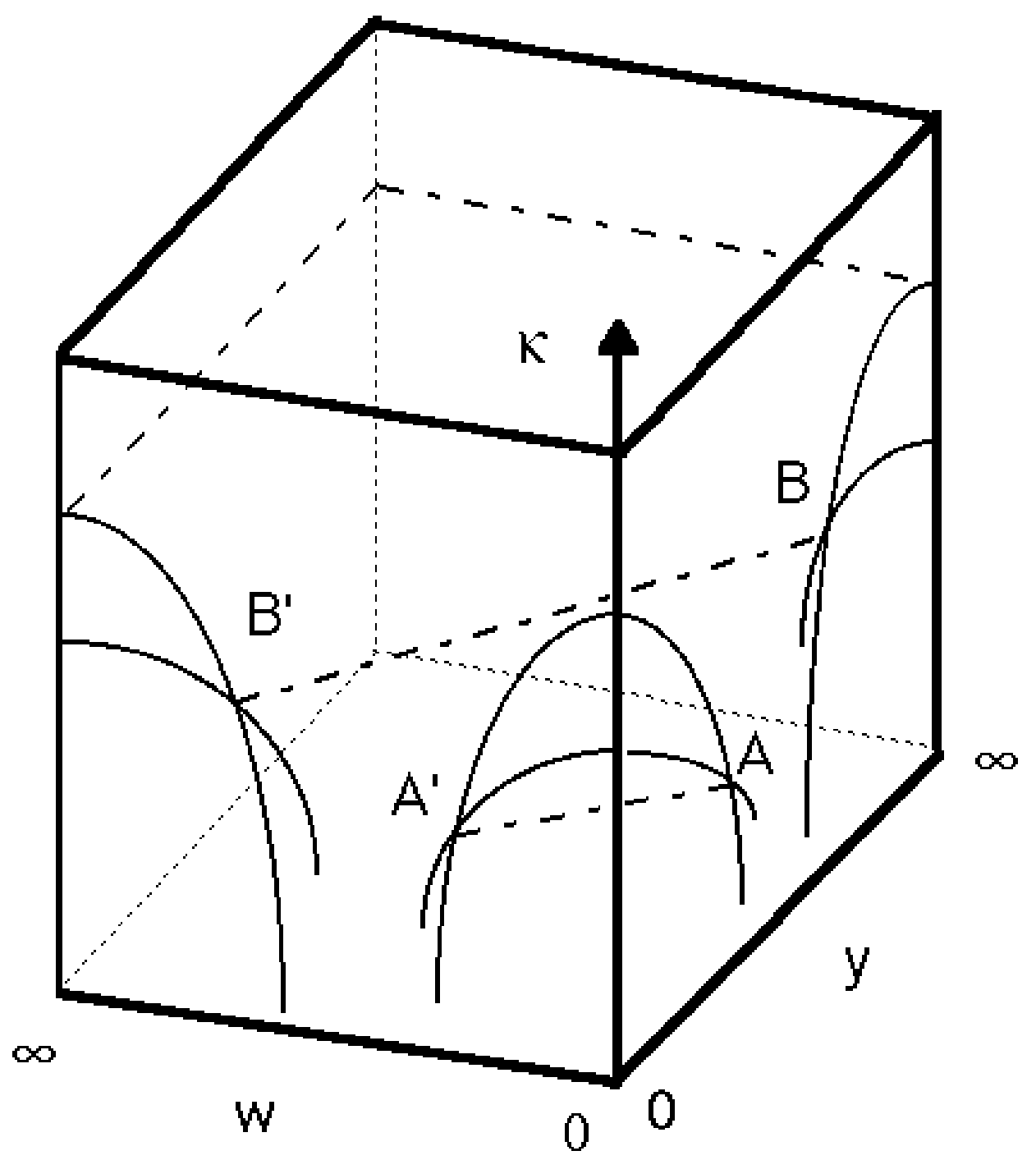

Fig. 2(a): Schematic three-dimensional phase diagram with the Wilson-Yukawa term. 


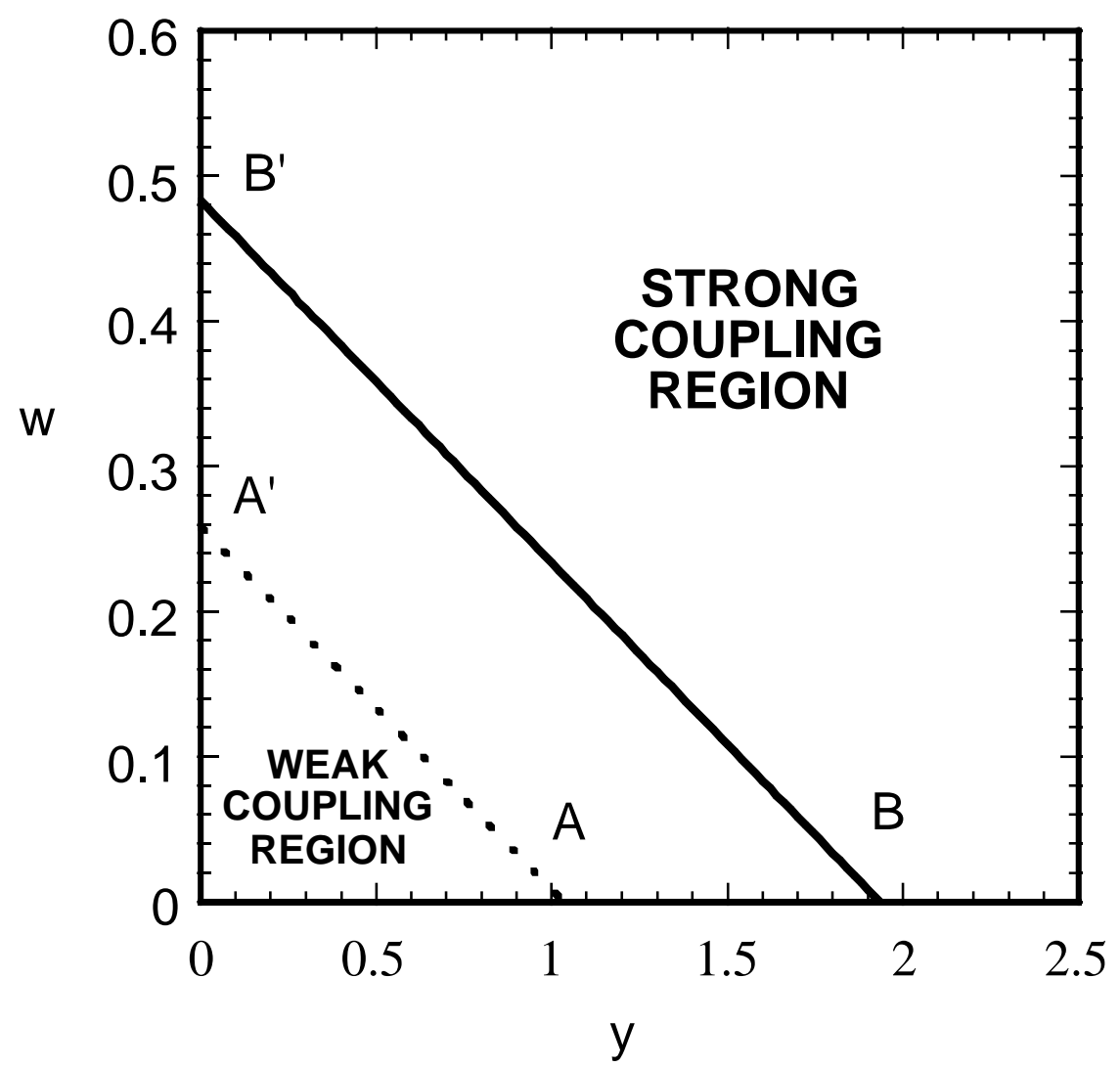

Fig. 2(b): Projection of the phase diagram on the $\kappa=$ constant plane. 